\title{
Malignant renal epithelioid angiomyolipoma: A case report and review of the literature
}

\author{
BAOYIN GUO ${ }^{1}$, HUALIN SONG ${ }^{2}$, JIULING YUE ${ }^{3}$ and GANG $\mathrm{LI}^{2}$ \\ ${ }^{1}$ Department of Urology, Tianjin Baodi Hospital of Tianjin Medical University, Tianjin 300321; \\ ${ }^{2}$ Department of Urology, The Second Hospital of Tianjin Medical University, Tianjin Institute of Urology, Tianjin 300211; \\ ${ }^{3}$ Department of Head and Neck Surgery, Tianjin Medical University Cancer Institute and Hospital, Tianjin 300060, P.R. China
}

Received November 24, 2014; Accepted September 28, 2015

DOI: $10.3892 / 01.2015 .3846$

\begin{abstract}
Malignant renal epithelioid angiomyolipoma (EAML) is rare, and currently there is no malignant criteria for its pathological diagnosis. In the present study, the case of a patient who suffered malignant renal EAML and underwent nephrectomy is reported. The histological patterns of the tumor were composed of sheets or nests of large polygonal epithelioid cells and thick-walled blood vessels, with clear mitoses. Immunohistochemistry demonstrated that the epithelioid and smooth muscle cells characteristically expressed human melanoma black-45, epithelial membrane antigen and actin. Pathological evaluation revealed malignant EAML with regional lymph node metastases. Magnetic resonance imaging and X-ray examination identified multiple liver and lung nodules at 16 months post-surgery. Since the patient did not respond to the initial treatment with doxorubicin and cisplatin, sorafenib was subsequently administered. However, the treatment was not effective, and the patient succumbed to multiple metastases six months later.
\end{abstract}

\section{Introduction}

Epithelioid angiomyolipoma (EAML) is an independent subtype of tumor within the family of perivascular epithelioid cell tumors (PEComas) (1). EAML is a rare variant of renal AML with malignant potential, and is characterized by a predominance of human melanoma black (HMB)-45 epithelioid cells and absence of adipocytes (2). Malignant EAML has been recently described as a rare tumor of the kidney, although its existence had been previously questioned (3). Several cases of EAML metastasized to the liver, lung and bone have been reported in the literature $(4,5)$. In the present

Correspondence to: Dr Gang Li, Department of Urology, The Second Hospital of Tianjin Medical University, Tianjin Institute of Urology, 23 Pingjiang Road, Tianjin 300211, P.R. China

E-mail: gangli797980@163.com

Key words: malignant tumor, epithelioid angiomyolipoma, kidney, neoplasm metastasis report, the case of a malignant renal EAML is described. The diagnosis was established by histological findings, and confirmed by the presence of multiple metastases. The diagnosis of EAML may be challenging, due to the similarity of its epithelioid morphology with that of renal cell carcinoma (RCC) (6). Therefore, an awareness of this entity and its characteristic features, including immunoreactivity with HMB-45, may aid its identification. Furthermore, the classification criterion for this malignancy proposed in the present study may contribute to understanding the pathological findings and clinical behavior of malignant renal EAML. The family of the patient provided written informed consent.

\section{Case report}

A 48-year-old woman with a history of flank pain in the right-side for $\sim 10$ days presented to the Department of Urology of Tianjin Baodi Hospital of Tianjin Medical University (Tianjin, China). Physical examination identified a palpable tender mass in the right flank. The patient did not present gross hematuria. No history of tuberous sclerosis syndrome (TSC) was recorded for the patient or her family, and the past medical history of the patient was not contributory. The results of routine laboratory tests, including kidney function, were normal, with the exception of macroscopic hematuria. Ultrasound scan revealed a mass arising from the lower pole of the right kidney, which did not display a typical adipose ultrasonographic echo. Computed tomography (CT) was performed using a SOMATOM Definition double-source helical scanner CT (Siemens, Medical Systems, Germany) and detected a soft tissue mass of heterogeneous density, which measured $\sim 13 \times 12 \times 11 \mathrm{~cm}$ and occupied the majority of the right kidney. In addition, several enlarged lymph nodes were noticed in the retroperitoneal space, with the biggest node measuring $\sim 3 \mathrm{~cm}$ in diameter (Fig. 1). The majority of the right renal pelvis and normal renal parenchyma structures were destroyed, and no obvious adipose tissues were detected. Scattered necrosis and calcification were occasionally observed, and the lesion exhibited moderate heterogeneous enhancement during enhanced scanning (Fig. 2). Radiographically, the mass was considered to be RCC. During surgery, several enlarged lymph nodes were identified, in addition to a large mass in the right 


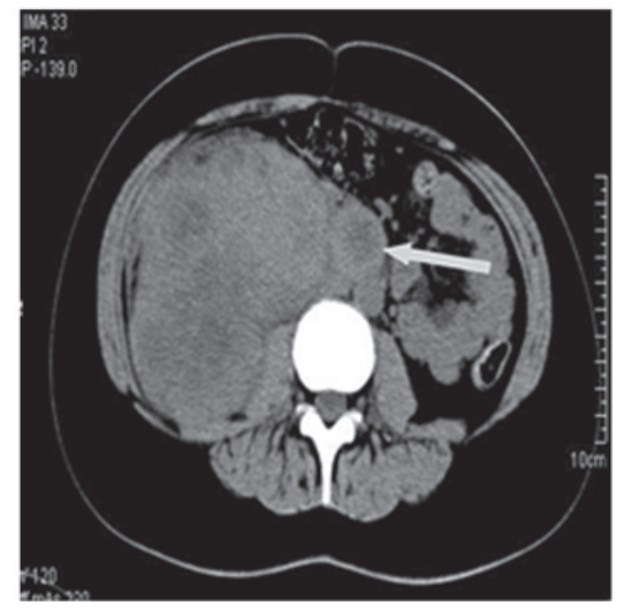

Figure 1. Axial precontrast computed tomography images demonstrating a large mass with heterogeneous density, measuring $13 \times 12 \times 11 \mathrm{~cm}$ in size, which was located at the lower pole of the right kidney. In addition, an enlarged lymph node of $3 \mathrm{~cm}$ in diameter, was also noted (white arrow).

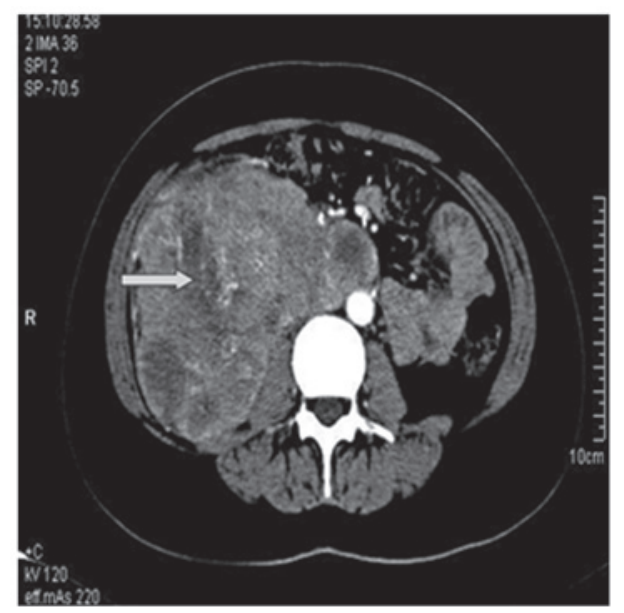

Figure 2. Postcontrast computed tomography images revealed moderately heterogeneous enhancement. The mass exhibited low density and irregular patchy attenuation, suggestive of necrosis (white arrow).

kidney with extension to the posterior peritoneum. The mass was contiguous with the mid-lower pole of the left kidney, without distinct surgical plane. The patient successfully underwent radical right nephrectomy with retroperitoneal lymphadenectomy, and experienced an uneventful recovery.

On histological examination, the specimen was brownish, and necrosis was observed. The tumor was composed of large polygonal epithelioid cells with abundant eosinophilic cytoplasm and markedly bizarre atypical nuclei, which were different from conventional AML (Fig. 3). While adipose tissue was scarcely observed, mitotic figures were often encountered, and atypical mitoses were also detected. Identical tumor cells were observed in the metastatic lymph nodes lesions and renal veins. The sections were immunostained using monoclonal anti-HMB-45 (no. SIG-3116; Covance, Inc., Princeton, NJ, USA), anti-desmin (no. ab919901; Abcam, Cambridge, UK) and anti-melan-A (ab187369; Abcam) and counterstained with hematoxylin and eosin (Abcam). Immunohistochemical staining confirmed that the epithelioid cells focally expressed

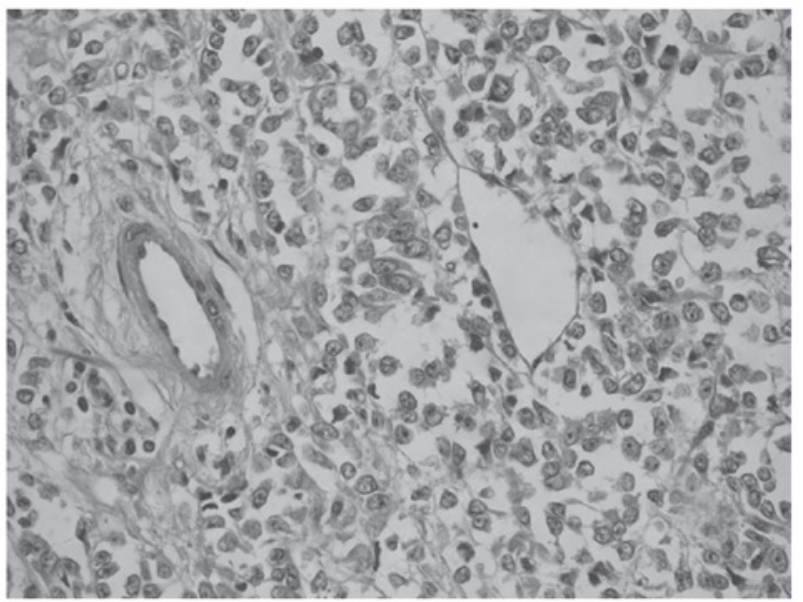

Figure 3. Microscopically, the tumor was composed of large polygonal epithelioid cells with markedly bizarre atypical mitotic nuclei, while adipose tissue was scarcely observed (hematoxylin and eosin stain; magnification, x200).

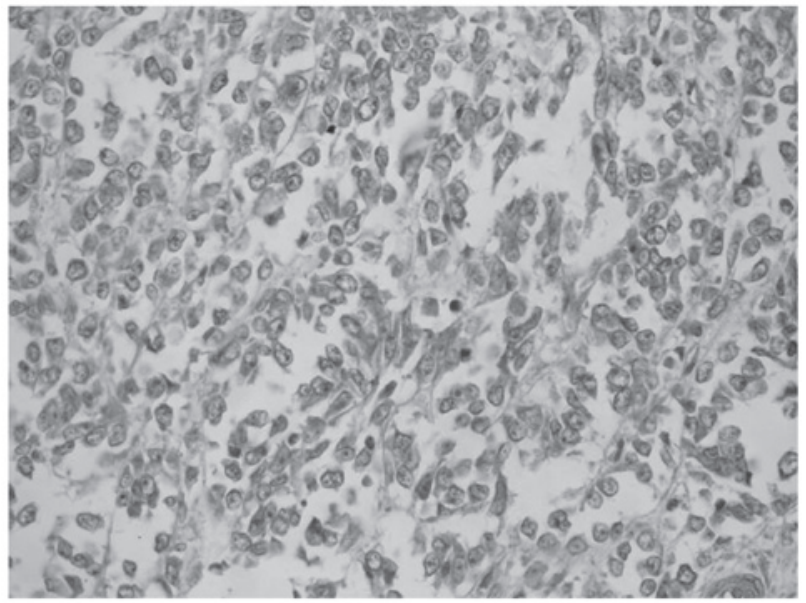

Figure 4. Immunohistochemical staining demonstrated that the epithelioid cells and spindle smooth muscle cells focally expressed human melanoma black-45 (magnification, x200).

HMB-45 (Fig. 4), and were moderately positive for melan-A and desmin. Thus, the pathological diagnosis was EAML with malignant tendency. Postoperative abdominal magnetic resonance imaging and chest X-ray scan conducted at 16 months of follow-up identified multiple liver and lung metastases. A fine-needle aspiration of the liver demonstrated the presence of EAML, which was morphologically and immunohistochemically identical to the primary renal tumor, thus confirming the existence of malignant EAML. In consequence, the patient was subjected to chemotherapy. However, no reduction in the size of the tumors was observed following two cycles of chemotherapy with doxorubicin and cisplatinum. The patient was then advised to participate in a clinical trial (enrolment no. 33567; 2013-12-8) studying the effects of sorafenib in the treatment of renal malignant tumors. The patient received two doses of $400 \mathrm{mg}$ sorafenib for two months. However, the treatment was not effective, and the metastatic lesions became more aggressive, extending to the liver, lungs and bones. The patient succumbed to neoplastic progression of the disease six months later. 


\section{Discussion}

EAML has been recognized as a mesenchymal tumor in the 2004 World Health Organization classification of renal tumors (7), and a member of the family of PEComas (8), which may occur sporadically or in association with TSC (9). EAML is considered a potentially malignant neoplasm, since $\sim$ a third of the reported cases of EAML developed metastatic lesions (10). Radiologically, the diagnosis of malignant EAML is difficult. While renal AML may be detected by CT, due to its adipose content, malignant EAML may be difficult to diagnose, due to the low abundance of adipocytes in this tumor (11). Upon review of the current literature, a limited number of imaging studies on malignant EAML were identified $(12,13)$. A previous study reported the diagnosis of a case of AML without fat density on CT (14). Therefore, fat density does not appear to be crucial for distinguishing malignant EAML from AML. In the present case, CT identified a renal mass that exhibited central low attenuation consistent with necrosis, in addition to multiple enlarged retroperitoneal lymph nodes. This CT appearance was similar to typical RCC (15), therefore raising the suspicion of malignancy. Malignant tumors often share certain common features, including relatively large size, heterogeneous attenuation, irregular contour, necrosis and multiple metastases (11). Multicentric EAML may be difficult to differentiate from metastasis in the presence of tumor thrombus in the inferior vena cava or in the adjacent organs, or when invasion of lymph nodes or blood vessels exists (16). The imaging features of the tumor in the present case included large size with necrosis on non-contrast CT, markedly heterogeneous enhancement, and regional lymph node metastases. Radiologically, the diagnosis suggested the possibility of malignancy. Therefore, awareness of these radiological findings may facilitate the detection of malignant EAML. Further studies on the imaging features of EAML will be required in the future.

The pathogenesis and mechanisms of the malignant transformation of EAML remain unclear. The optimal criteria for the diagnosis of malignant EAML may require to be reassessed, since necrosis, hemorrhage, nuclear atypia and mitotic activity are considered to indicate a potentially malignant tumor. However, there are no histological criteria for malignant EAML, with the exception of distant metastases, which are accepted to be a definite sign of malignancy. In the present case, the tumor was demonstrated to be malignant due to the occurrence of distant metastases. Histologically, EAML is characterized by the presence of predominantly polygonal epithelioid cells with atypical nuclei, mitotic figures, necrosis, marked atypical large cells with abundant eosinophilic cytoplasm that stain strongly for HMB-45, and low number of adipose cells (17). Therefore, malignant EAML should be carefully differentiated from RCC, and confirmed by pathology and immunohistochemistry. Additionally, a previous study has indicated that Ki-67 may be a useful marker in the diagnosis of malignant EAML (18).

Malignant EAML is aggressive and may be life-threatening (19). Therefore, the clinicians should be aware of it, and consider EAML as a potential malignant disease. Currently, there is no known effective therapy for malignant EAML other than surgery (20). Although nephron sparing surgery is an alternative treatment for malignant renal EAML, local recurrence and metastasis have been reported following surgery (10). Surgical removal of metastases often contributes to good prognosis (20). Due to the difficulty in differentiating malignant EAML from RCC, nephrectomy is a common procedure for large EAMLs. However, nephrectomy alone may be inadequate in certain cases, and adjuvant therapy should be considered (21).

Chemotherapy for malignant EAML is still under debate, although a number of patients with EAML have been reported to respond to doxorubicin (10). However, the efficacy of this treatment is not clear at present, despite the fact that certain cases exhibited good response at the beginning of the treatment. In addition the long-term effects associated with doxorubicin have not been determined thus far. In a recent study, Kenerson et al (22) reported an EAML mass that uniformly exhibited activation of the mechanistic target of rapamycin (mTOR) cascade, which suggests that mTOR inhibitors may provide a therapeutic benefit in the treatment of EAML. Multimodal treatments for malignant EAML, which consist of chemotherapeutic and molecular targeted agents, have been proposed (23). However, this type of approach was not successful in the present case, since the patient did not respond to chemotherapy in combination with sorafenib.

Malignant EAML presents a relatively favorable prognosis without relapse or metastasis following surgery. However, patients with remote organ metastases, mainly in the lungs and liver, present relatively poor prognosis, and require a closer follow-up of these tumors.

\section{References}

1. Thway K and Fisher C: PEComa: Morphology and genetics of a complex tumor family. Ann Diagn Pathol 19: 359-368, 2015.

2. Konosu-Fukaya S, Nakamura Y, Fujishima F, et al: Renal epithelioid angiomyolipoma with malignant features: Histological evaluation and novel immunohistochemical findings. Pathol Int 64: 133-141, 2014.

3. Mahdi Y, Znati K, Iken A, et al: Malignant renal epithelioid angiomyolipoma associated with abdominopelvic hydatid cysts: A case report. J Med Case Rep 10: 80, 2015.

4. Huang KH, Huang CY, Chung SD, Pu YS, Shun CT and Chen J: Malignant epithelioid angiomyolipoma of the kidney. J Formos Med Assoc 106 (Suppl): S51-S54, 2007.

5. Lau SK, Marchevsky AM, McKenna RJ Jr and Luthringer DJ: Malignant monotypic epithelioid angiomyolipoma of the retroperitoneum. Int J Surg Pathol 11: 223-228, 2003.

6. Svec A and Velenská Z: Renal epithelioid angiomyolipoma - a close mimic of renal cell carcinoma. Report of a case and review of the literature. Pathol Res Pract 200: 851-856, 2005.

7. Lopez-Beltran A, Scarpelli M, Montironi R and Kirkali Z: 2004 WHO classification of the renal tumors of the adults. Eur Urol 49: 798-805, 2006

8. Martignoni G, Pea M, Reghellin D, Zamboni G and Bonetti F: PEComas: The past, the present and the future. Virchows Arch 452: 119-132, 2008.

9. Aydin H, Magi-Galluzzi C, Lane BR, Sercia L, Lopez JI, Rini BI and Zhou M: Renal angiomyolipoma: Clinicopathologic study of 194 cases with emphasis on the epithelioid histology and tuberous sclerosis association. Am J Surg Pathol 33: 289-297, 2009.

10. Cibas ES, Goss GA, Kulke MH, Demetri GD and Fletcher CD: Malignant epithelioid angiomyolipoma ('sarcoma ex angiomyolipoma') of the kidney: A case report and review of the literature. Am J Surg Pathol 25: 121-126, 2001.

11. Cui L, Hu XY, Gong SC, Fang XM, Lerner A and Zhou ZY: A massive renal epithelioid angiomyolipoma with multiple metastatic lymph nodes. Clin Imaging 35: 320-323, 2011.

12. Radin R and Ma Y: Malignant epithelioid renal angiomyolipoma in a patient with tuberous sclerosis. J Comput Assist Tomogr 25: 873-875, 2001

13. El Jack AK, Tomaszewski JE, Haller DG and Siegelman ES: Metastatic PEComa arising from renal angiomyolipoma: MRI findings. J Magn Reson Imaging 26: 159-161, 2007. 
14. Nepple KG, Bockholt NA, Dahmoush L and Williams RD: Giant renal angiomyolipoma without fat density on CT scan: Case report and review of the literature. ScientificWorldJournal 10: 1334-1338, 2010.

15. Tsai CC, Wu WJ, Li CC, Wang CJ, Wu CH and Wu CC: Epithelioid angiomyolipoma of the kidney mimicking renal cell carcinoma: A clinicopathologic analysis of cases and literature review. Kaohsiung J Med Sci 25: 133-140, 2009.

16. Inci O, Kaplan M, Yalcin O, Atakan IH and Kubat H: Renal angiomyolipoma with malignant transformation, simultaneous occurrence with malignity and other complex clinical situations. Int Urol Nephrol 38: 417-426, 2006.

17. Tan G, Liu L, Qiu M, Chen L, Cao J and Liu J: Clinicopathologic features of renal epithelioid angiomyolipoma: Report of one case and review of literatures.Int J Clin Exp Pathol 8: 1077-1080, 2015.

18. Ooi SM, Vivian JB and Cohen RJ: The use of the Ki-67 marker in the pathological diagnosis of the epithelioid variant of renal angiomyolipoma. Int Urol Nephrol 41: 559-565, 2009.

19. Luo J, Liu B, Wang Y, Li J, Wang P, Chen J and Wang C: Comprehensive clinical and pathological analysis of aggressive renal epithelioid angiomyolipoma: report of three cases. Onco Targets Ther 27: 823-827, 2014.
20. Vicens RA, Jensen CT, Korivi BR and Bhosale PR: Malignant renal epithelioid angiomyolipoma with liver metastasis after resection: a case report with multimodality imaging and review of the literature. J Comput Assist Tomogr 38: 574-577, 2014.

21. Varma S, Gupta S, Talwar J, Forte F and Dhar M: Renal epithelioid angiomyolipoma: A malignant disease. J Nephrol 24: $18-22,2011$.

22. Kenerson H, Folpe AL, Takayama TK and Yeung RS: Activation of the mTOR pathway in sporadic angiomyolipomas and other perivascular epithelioid cell neoplasms. Hum Pathol 38: 1361-1371, 2007.

23. Allegra A, Coppolino G, Bolignano D, Giacobbe MS, Alonci A D'Angelo A, Bellomo G, Teti D, Loddo S, Musolino C and Buemi M: Endothelial progenitor cells: Pathogenetic role and therapeutic perspectives. J Nephrol 22: 463-475, 2009. 